\title{
A Multi-Antenna Detection Algorithm for UMTS/TDD Receivers in Strong Interference Environments
}

\author{
Harold Artés, Klaus Kopsa, and Franz Hlawatsch \\ Institute of Communications and Radio-Frequency Engineering, Vienna University of Technology \\ Gusshausstrasse 25/389, A-1040 Vienna, Austria \\ phone: +43 158801 38923, fax: +43 158801 38999, email: harold.artes@nt.tuwien.ac.at \\ web: http://www.nt.tuwien.ac.at/dspgroup/time.html
}

\begin{abstract}
We propose a space-time detection algorithm for a UMTS/TDD downlink scenario with strong interference. Conventional space-time detection schemes based on MMSE equalization require accurate estimation of the correlation matrix of the received signal vector, which is difficult because of the time variation of the mobile radio channel. We thus develop a decision feedback detection scheme in which the correlation matrix is "detected" rather than estimated. For detection of the active data channels, a maximal invariant detection statistic is presented. Simulation results demonstrate that the proposed space-time detection algorithm features good performance for various realistic propagation scenarios even at low SINR.
\end{abstract}

\section{INTRODUCTION}

As third-generation systems are being deployed, network operators require accurate measurement tools to assess the interference situation present in their network. Within the European IST project ANTIUM [1], we develop off-line multiantenna signal processing algorithms for analyzing the strength and origin of interfering signals in a UMTS/TDD network. Since the ANTIUM equipment is not actively connected to the monitored network, only the downlink signals transmitted by the different base stations (BSs) are available. We thus attempt to demodulate the broadcast channels (BCHs) of surrounding BSs and extract the cell IDs. This information allows network operators to quantify how different BSs contribute to the total interference and, in turn, to adjust their network accordingly.

The overall demodulation procedure consists of synchronization [2], channel estimation [3], and detection. In this paper, we present a multi-antenna decision feedback algorithm for detection of the $\mathrm{BCHs}$ of surrounding $\mathrm{BSs}$ in a strong interference scenario. Our algorithm uses a detection scheme for determination of the correlation matrix of the received signal. This approach yields good receiver performance even at low SINR and for a fast fading channel.

The paper is organized as follows. The UMTS/ TDD system layout is reviewed in the remainder of this section. Section II discusses the channel model used. The decision feedback detection algorithm is presented in Sections III through V.

Funding by EU-IST project ANTIUM and FWF grant P15156-N02.

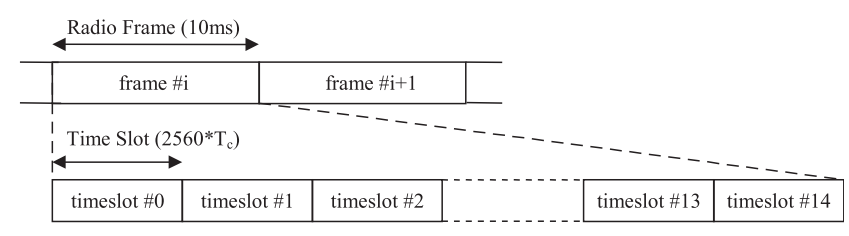

Fig. 1. Physical channel signal format [4].

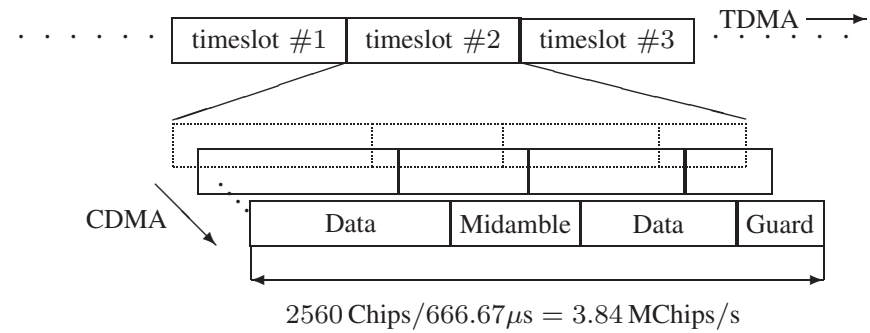

Fig. 2. Structure of a UMTS/ TDD timeslot [4].

Finally, in Section VI, the performance of our algorithm is assessed through simulation.

UMTS/TDD Timeslot Structure. UMTS/TDD [4] uses timedivision-duplex (TDD), i.e., a timeslot structure, to separate uplink and downlink. In every timeslot, code-division multiple access (CDMA) is used. Each UMTS/TDD radio frame contains 15 timeslots (see Fig. 1) that can be individually allocated for uplink or downlink in a flexible manner. Only one of these 15 timeslots contains the desired $\mathrm{BCH}$ along with some synchronization information.

As shown in Fig. 2, each timeslot consists of two data parts separated by a midamble and followed by a guard period. In the timeslot containing the $\mathrm{BCH}$, the two data parts consist of the $\mathrm{BCH}$ and up to 12 additional data channels. Each individual channel is spread using an associated spreading code with spreading factor 16 [5]. The sum of the spread channels is then scrambled (modulated, see [5]) with a cell-specific scrambling code of length 16. Finally, the resulting signal is transmitted over a frequency-selective fading channel and received by the $M$-element antenna array of the mobile receiver. 


\section{Channel Model}

The channel model used here [6-8] is based on the following assumptions. Every BS has one transmit antenna and the receiver has $M$ receive antennas. Synchronization [2] and channel estimation [3] have been performed previously, and the synchronization signal of all BSs has been cancelled. Due to the fact that the entire UMTS/TDD network is synchronized, the BCHs of all BSs are transmitted in the same timeslot and hence it suffices to consider only this timeslot in the following.

Let $U$ denote the number of surrounding BSs (known from the synchronization stage) and let the index set $\mathcal{L}^{(u)} \subseteq$ $\{5, \cdots, 16\}$ denote the set of active data channels for the $u$ th $\mathrm{BS}$. (Index 1 corresponds to the $\mathrm{BCH}$ that is always active, and indices 2, 3, and 4 are never active for the timeslot of interest.) The $k$ th data channel transmitted by the $u$ th $\mathrm{BS}$ (here, $k \in \mathcal{L}^{(u)}$ and $u \in\{1, \cdots, U\}$ ) contains 976 chips and thus $976 / 16=61$ CDMA symbols. We will combine these 61 CDMA symbols and the 61 CDMA symbols of the second data part contained in the same timeslot (cf. Fig. 2) into the vector $\mathbf{d}_{k}^{(u)} \triangleq\left(d_{k}^{(u)}[1] \cdots d_{k}^{(u)}[122]\right)^{T}$. The data symbols $\mathbf{d}_{k}^{(u)}$ are spread by the data-channel-specific spreading code $c_{k}[n]$ and scrambled by the BS-dependent scrambling code $s^{(u)}[n]$, both of length 16 . The scrambling code is known from the synchronization step [2].

Let $h^{(u, m)}[n]$ denote the (equivalent discrete-time, baseband) impulse response of the mobile radio channel from the $u$ th $\mathrm{BS}$ to the $m$ th receive antenna, with $n$ a chip-rate time index, and let $L-1$ denote the maximum delay (i.e., $h^{(u, m)}[n]=0$ for $n \notin\{0, \cdots, L-1\}$ for all $\left.u, m\right)$. We assume all channels to be time-invariant during the transmission of one frame. The $h^{(u, m)}[n]$ are known from the channel estimation step [3]. The composite impulse response associated to the $k$ th data channel, $u$ th $\mathrm{BS}$, and $m$ th receive antenna is given by

$a_{k}^{(u, m)}[n] \triangleq\left(h^{(u, m)} * c_{k}^{(u)}\right)[n], \quad$ with $c_{k}^{(u)}[n] \triangleq s^{(u)}[n] c_{k}[n]$,

where $*$ denotes discrete-time convolution. Let $\mathbf{a}_{k}^{(u, m) \triangleq}$ $\left(a_{k}^{(u, m)}[1] \cdots a_{k}^{(u, m)}[16+L-1]\right)^{T}$. The signal component received at the $m$ th antenna that is due to the $k$ th data channel from the $u$ th BS can be written as $\mathbf{x}_{k}^{(u, m)}=c_{k}^{(u)} \mathbf{A}_{k}^{(u, m)} \mathbf{d}_{k}^{(u)}$, with the $(122 \cdot 16+L-1) \times 1$ vector $\mathbf{x}_{k}^{(u, m)}$ and the $(122 \cdot 16+L-1) \times 122$ block-Toeplitz matrix

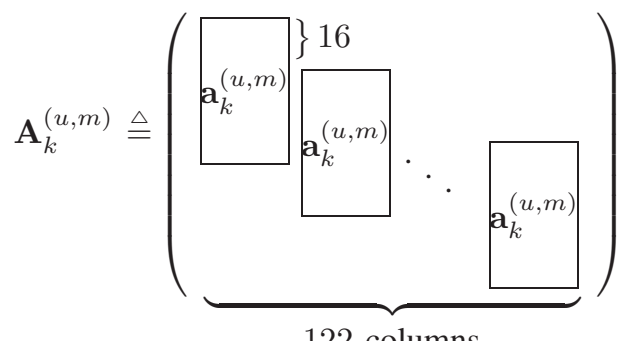

122 columns

(we note that the presence of the midamble is neglected in the definition of $\mathbf{A}_{k}^{(u, m)}$ ). The gain factor $c_{k}^{(u)}$ is due to power control (however, $c_{1}^{(u)}=1$ because the $\mathrm{BCH}$ has no power control). The overall received signal at the $m$ th antenna is thus given by

$$
\mathbf{x}^{(m)}=\sum_{u=1}^{U}\left[\mathbf{A}_{1}^{(u, m)} \mathbf{d}_{1}^{(u)}+\sum_{k \in \mathcal{L}^{(u)}} c_{k}^{(u)} \mathbf{A}_{k}^{(u, m)} \mathbf{d}_{k}^{(u)}\right]+\mathbf{w}^{(m)},
$$

where $\mathbf{w}^{(m)}$ is a noise vector. Stacking all antenna signal vectors as $\mathbf{x} \triangleq\left(\mathbf{x}^{(1) T} \cdots \mathbf{x}^{(M) T}\right)^{T}$, we can write

$$
\mathbf{x}=\mathbf{A C d}+\mathbf{w},
$$

with the $M(122 \cdot 16+L-1) \times 122\left(U+\sum_{u=1}^{U-1}\left|\mathcal{L}^{(u)}\right|\right)$ matrix $\mathbf{A}=\left(\mathbf{A}_{\mathrm{BCH}}, \mathbf{A}_{\mathrm{DAT}}\right)$, where

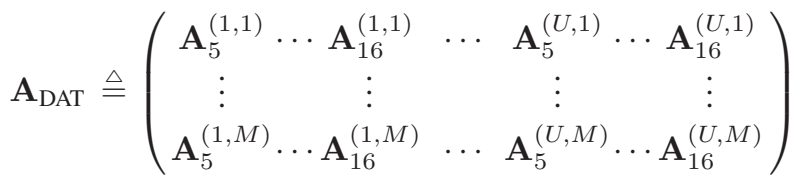

$$
\begin{aligned}
& \mathbf{A}_{\mathrm{BCH}} \triangleq\left(\begin{array}{ccc}
\mathbf{A}_{1}^{(1,1)} & \cdots & \mathbf{A}_{1}^{(U, 1)} \\
\vdots & & \vdots \\
\mathbf{A}_{1}^{(1, M)} & \cdots & \mathbf{A}_{1}^{(U, M)}
\end{array}\right) \text {, }
\end{aligned}
$$

the diagonal matrix $\mathbf{C}=\operatorname{diag}\left\{1, c_{5}^{(1)}, \cdots, c_{16}^{(1)}, \cdots, 1, c_{5}^{(U)}, \cdots\right.$, $\left.c_{16}^{(U)}\right\} \otimes \mathbf{I}_{122 \times 122}$ (i.e., each one of the numbers $1, c_{5}^{(1)}, \cdots, c_{16}^{(1)}$, $\cdots, 1, c_{5}^{(U)}, \cdots, c_{16}^{(U)}$ is repeated 122 times on the diagonal), and the vectors $\mathbf{w} \triangleq\left(\mathbf{w}^{(1) T} \ldots \mathbf{w}^{(M) T}\right)^{T}$ and $\mathbf{d} \triangleq\left(\mathbf{d}_{1}^{(1) T} \ldots\right.$ $\left.\mathbf{d}_{16}^{(1) T} \cdots \mathbf{d}_{1}^{(U) T} \cdots \mathbf{d}_{16}^{(U) T}\right)^{T}$. The above definitions of $\mathbf{A}_{\mathrm{DAT}}$, $\mathbf{C}$, and $\mathbf{d}$ apply if all data channels are active. In general, only the entries corresponding to active data channels appear in $\mathbf{A}_{\mathrm{DAT}}, \mathbf{C}$, and $\mathbf{d}$. Thus, whereas the submatrices $\mathbf{A}_{k}^{(u, m)}$ are known, it is unknown which of them are contained in $\mathbf{A}_{\mathrm{DAT}}$.

\section{A Space-Time Decision FeEdback Receiver}

A simple space-time detector would use MMSE equalization (e.g., [7]) followed by quantization. This receiver would require knowledge of the correlation matrix of the received signal $\mathbf{x}$,

$$
\mathbf{R}_{\mathbf{x}} \triangleq \mathbb{E}\left\{\mathbf{x x}^{H}\right\}=\mathbf{A} \mathbf{C} \mathbf{C}^{H} \mathbf{A}^{H}+\sigma_{w}^{2} \mathbf{I} .
$$

Accurate estimation of $\mathbf{R}_{\mathbf{x}}$ from the observed $\mathbf{x}$ is difficult because the channel changes from slot to slot. Unfortunately, $\mathbf{R}_{\mathbf{x}}$ has to be known with high accuracy because the data channels transmitted by the stronger BSs act as strong interferers for the BCHs of weaker BSs. As a result, the performance of the MMSE equalizer is unsatisfactory.

Therefore, instead of estimating $\mathbf{R}_{\mathbf{x}}$ from the received signal, we will adopt a strategy that is based on the expression (2). Calculation of $\mathbf{R}_{\mathbf{x}}$ according to (2) would presuppose detection of the sets $\mathcal{L}^{(u)}$ of active data channels for all BSs (in order to determine $\mathbf{A}_{\mathrm{DAT}}$ ) and estimation of the gain factor matrix C. Because this works best for the strongest BS, we propose a decision feedback (DFB) receiver that, initially, only takes the strongest BS into account. For this BS, we detect $\mathcal{L}^{(u)}$ and estimate $\mathbf{C}$ and then detect the part of $\mathbf{d}$ corresponding to the strongest $\mathrm{BS}$, i.e., the desired $\mathrm{BCH}$ and the interfering user data. Next, the component of the received 


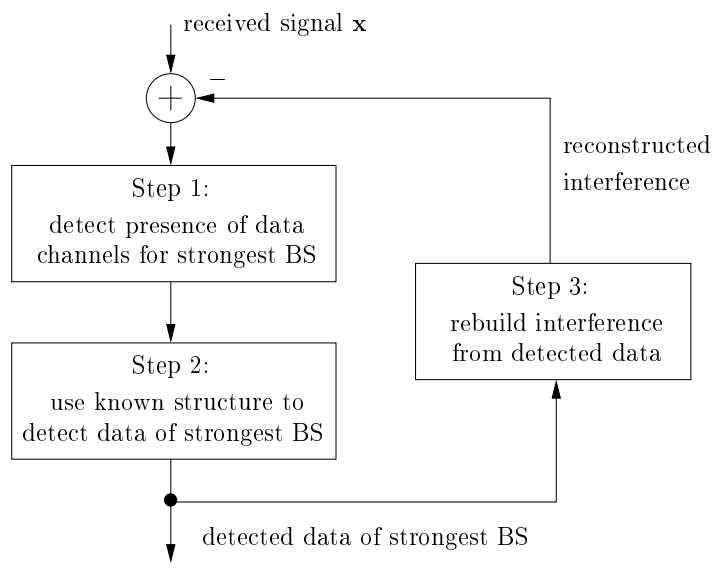

Fig. 3. Decision feedback detection scheme.

signal corresponding to this data is calculated and subtracted from the overall received signal. Then the whole procedure is repeated for the second strongest BS, etc. This recursive DFB (or interference cancellation) scheme is illustrated in Fig. 3.

Suppose that the index of the strongest BS is $u_{0}$. In the first step, we model the received signal as (cf. (1))

$$
\mathbf{x}=\mathbf{A}^{\left(u_{0}\right)} \mathbf{C}^{\left(u_{0}\right)} \mathbf{d}^{\left(u_{0}\right)}+\mathbf{w}
$$

where $\mathbf{A}^{\left(u_{0}\right)}=\left(\mathbf{A}_{\mathrm{BCH}}^{\left(u_{0}\right)}, \mathbf{A}_{\mathrm{DAT}}^{\left(u_{0}\right)}\right)$, with $\mathbf{A}_{\mathrm{BCH}}^{\left(u_{0}\right)}$ and $\mathbf{A}_{\mathrm{DAT}}^{\left(u_{0}\right)}$ containing only the entries $\mathbf{A}_{k}^{\left(u_{0}, m\right)}$, and $\mathbf{d}^{\left(u_{0}\right)}=\left(\mathbf{d}_{1}^{\left(u_{0}\right) T} \ldots\right.$ $\left.\mathbf{d}_{16}^{\left(u_{0}\right) T}\right)^{T}$. The vector $\mathbf{w}$ contains the noise and the interference from all other BSs and will be modeled as being uncorrelated for simplicity. We first calculate $\hat{\mathcal{L}}^{\left(u_{0}\right)}$ (from which we can calculate an estimate $\left.\hat{\mathbf{A}}^{\left(u_{0}\right)}\right)$ and $\hat{\mathbf{C}}^{\left(u_{0}\right)}$ by means of the methods to be explained in Sections IV and V. Using an MMSE equalization approach, the detected data in the first step is then given by $\hat{\mathbf{d}}^{\left(u_{0}\right)}=Q\{\mathbf{y}\}$, where $Q\{\cdot\}$ denotes componentwise quantization according to the QPSK symbol alphabet used and

$\mathbf{y}=\left(\hat{\mathbf{C}}^{\left(u_{0}\right) H} \hat{\mathbf{A}}^{\left(u_{0}\right) H} \hat{\mathbf{A}}^{\left(u_{0}\right)} \hat{\mathbf{C}}^{\left(u_{0}\right)}+\widehat{\sigma_{w}^{2}} \mathbf{I}\right)^{-1} \hat{\mathbf{C}}^{\left(u_{0}\right) H} \hat{\mathbf{A}}^{\left(u_{0}\right) H} \mathbf{x}$

is the result of MMSE equalization based on the model (3) (here, $\widehat{\sigma_{w}^{2}}$ is an estimate of the interference-plus-noise variance). Subsequently, the component of the received signal corresponding to the detected data $\hat{\mathbf{d}}^{\left(u_{0}\right)}$ is calculated according to the model (3) and subtracted from the overall received signal:

$$
\tilde{\mathbf{x}}=\mathbf{x}-\hat{\mathbf{A}}^{\left(u_{0}\right)} \hat{\mathbf{C}}^{\left(u_{0}\right)} \hat{\mathbf{d}}^{\left(u_{0}\right)} .
$$

This procedure is repeated using $\tilde{\mathbf{x}}$ instead of $\mathbf{x}$ and the second strongest BS instead of the strongest one, etc.

\section{Detection of Data Channels}

We will now propose a scheme for calculating $\hat{\mathcal{L}}^{(u)}$ (for simplicity, the index of the strongest BS is now denoted by $u$ instead of $\left.u_{0}\right)$. Consider the vector $\mathbf{x}[i] \triangleq\left(\left(\mathbf{x}^{(1)}\right)_{i \cdot 16} \cdots\right.$ $\left.\left(\mathbf{x}^{(1)}\right)_{i \cdot 16+15} \cdots\left(\mathbf{x}^{(M)}\right)_{i \cdot 16} \cdots\left(\mathbf{x}^{(M)}\right)_{i \cdot 16+15}\right)^{T}$ of length $16 M$. This vector contains the components from all active data channels $k \in \mathcal{L}^{(u)}$ from the strongest BS $u$ at symbol time $i$. Assuming for simplicity that $\mathbf{C}=\mathbf{I}$ (i.e., no power control; see Subsection IV-C for comments on the case of power control), we can write

$$
\mathbf{x}[i]=\sum_{k \in \mathcal{L}^{(u)}} d_{k}^{(u)}[i] \tilde{\mathbf{a}}_{k}^{(u)}+\mathbf{w}[i] .
$$

Here, $\tilde{\mathbf{a}}_{k}^{(u)} \triangleq\left(\tilde{\mathbf{a}}_{k}^{(u, 1) T} \ldots \tilde{\mathbf{a}}_{k}^{(u, M) T}\right)^{T}$ where $\tilde{\mathbf{a}}_{k}^{(u, m)}$ is a length16 vector whose elements equal the first 16 elements of $\mathbf{a}_{k}^{(u, m)}$, i.e., $\left(\tilde{\mathbf{a}}_{k}^{(u, m)}\right)_{l}=\left(\mathbf{a}_{k}^{(u, m)}\right)_{l}$ for $l=1, \cdots, 16$. Furthermore, $\mathbf{w}[i]$ accounts for the $\mathrm{BCH}$, contributions from weaker BSs, inter-symbol interference (ISI) from neighboring CDMA symbols, and noise. The expression (5) is valid for $i \in \mathcal{I}_{\text {data }}$, where $\mathcal{I}_{\text {data }}$ denotes the data-part interval (see Fig. 2). For simplicity, we will model $\mathbf{w}[i]$ as white, uncorrelated, and complex Gaussian. We also assume that the vectors $\tilde{\mathbf{a}}_{k}^{(u)}$ for different $k \in \mathcal{L}^{(u)}$ are orthogonal, so that the presence of each data channel can be detected individually rather than jointly.

\section{A. Incoherent Matched Filter}

For the model in (5), the incoherent matched filter [9] leads to the following detection statistic for the presence of the $k$ th data channel:

$$
\Lambda_{k}=\sum_{i \in \mathcal{I}_{\text {data }}}\left|T_{k}[i]\right|^{2}, \quad \text { with } T_{k}[i] \triangleq \mathbf{x}^{H}[i] \tilde{\mathbf{a}}_{k}^{(u)} .
$$

The detected index set $\hat{\mathcal{L}}^{(u)}$ then is defined as the set of indices $k \in\{5, \cdots, 16\}$ for which $\Lambda_{k}$ exceeds a certain threshold $\eta$. The choice of $\eta$ will be discussed in Subsection IV-C.

The incoherent matched filter is based on the assumption that the nuisance parameters $d_{k}^{(u)}[i]$ are circularly symmetric (i.e., rotationally invariant) complex Gaussian. However, the $d_{k}^{(u)}[i]$ are taken from a QPSK alphabet, and thus they are discrete random variables whose statistics is invariant only to rotations by multiples of $\pi / 2$.

\section{B. Detector Based on Maximal Invariant Statistic}

We now propose a detector that is invariant to rotations of the $d_{k}^{(u)}[i]$ by multiples of $\pi / 2$. Consider the statistic

$$
T_{k}^{\prime}[i] \triangleq\left|T_{k}[i]\right| e^{j \arg \bmod \left\{T_{k}[i]\right\}}
$$

where $T_{k}[i]=\mathbf{x}^{H}[i] \tilde{\mathbf{a}}_{k}^{(u)}$ as before and $\arg \bmod \{c\} \triangleq$ $[(\arg \{c\}+\pi / 4)$ modulo $\pi / 2]-\pi / 4$, i.e., the phase of the complex number $c$ is wrapped to the interval $[-\pi / 4, \pi / 4)$. It can be shown that $T_{k}^{\prime}[i]$ constitutes a maximal invariant statistic [10], which means that $T_{k}^{\prime}[i]$ is invariant to rotation of $d_{k}^{(u)}[i]$ by $\pi / 2$ and $\left\{T_{k}^{\prime}[i]\right\}_{i \in \mathcal{I}_{\text {data }}}$ contains all information relevant to our detection problem.

Fig. 4(a) shows the probability density function (pdf) of the statistic $T_{k}[i]$. Under hypothesis $\mathcal{H}_{0}$ (signal not present), the pdf of $T_{k}[i]$ is a 2-D Gaussian centered at the origin. Under hypothesis $\mathcal{H}_{1}$ (signal present), the pdf of $T_{k}[i]$ (conditional on the data symbol) is a 2-D Gaussian located about $1, j$, -1 , or $-j$ that is labeled $\mathcal{H}_{1}^{(1)}$ through $\mathcal{H}_{1}^{(4)}$ in Fig. 4(a). The dashed arrows show how the phase of $T_{k}[i]$ is wrapped 


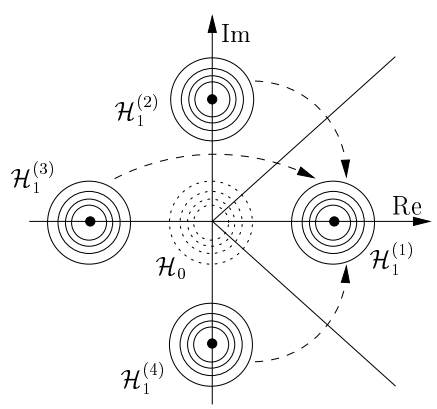

(a)

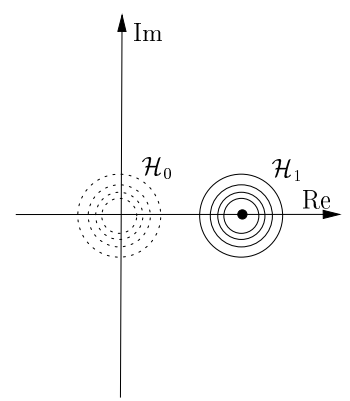

(b)
Fig. 4. Illustration of the maximal invariant statistic $T_{k}^{\prime}[i]$ : (a) Conditional pdf of $T_{k}[i]$, (b) approximate conditional pdf of $T_{k}^{\prime}[i]$ for high SNR. The dashed arrows in (a) show how the phase of $T_{k}[i]$ is wrapped to the fundamental phase interval $[-\pi / 4, \pi / 4)$ when $T_{k}[i]$ is converted to $T_{k}^{\prime}[i]$.

to the fundamental phase interval $[-\pi / 4, \pi / 4)$ through the transition from $T_{k}[i]$ to $T_{k}^{\prime}[i]$. Note, however, that only the pdf parts outside the sector $[-\pi / 4, \pi / 4)$ are transferred into $[-\pi / 4, \pi / 4)$; the "tails" of the pdf components $\mathcal{H}_{1}^{(2)}, \mathcal{H}_{1}^{(3)}$, and $\mathcal{H}_{1}^{(4)}$ that are located in $[-\pi / 4, \pi / 4]$ are left unchanged.

Ignoring these pdf tails, which is justified if the SNR is sufficiently high, we obtain an approximation to the conditional pdf of $T_{k}^{\prime}[i]$ that is depicted in Fig. 4(b). This approximate pdf corresponds to the standard problem of detecting a known signal in white Gaussian noise [9]. We thus arrive at the following detection statistic based on the maximal invariant statistic $\left\{T_{k}^{\prime}[i]\right\}_{i \in \mathcal{I}_{\text {data }}}$ :

$$
\Lambda_{k}^{\mathrm{inv}}=\sum_{i \in \mathcal{I}_{\text {data }}} \operatorname{Re}\left\{T_{k}^{\prime}[i]\right\} .
$$

\section{ROCs and Choice of Threshold}

Fig. 5(a) shows simulated receiver operating characteristics (ROCs) of the two detection statistics $\Lambda_{k}$ and $\Lambda_{k}^{\text {inv }}$ for a flat Rayleigh fading channel (note that there is no ISI) with 8 active and 4 inactive data channels, one BS, and white Gaussian noise with two different SNR values. For SNR $=-35 \mathrm{~dB}$, both ROCs are indistinguishable from 1 and thus both detection statistics yield detection probability $\approx 1$ even at false alarm probabilities smaller than 0.01 . For $\mathrm{SNR}=-40 \mathrm{~dB}$, the ROCs still are roughly equal but the detection probability is lower than 1 , i.e., there is now a clear tradeoff between low false alarm probability and high detection probability.

For an analysis of the influence of the threshold $\eta$ on the detection results, it is advantageous to normalize the detection statistics $\Lambda_{k}$ and $\Lambda_{k}^{\text {inv }}$ such that, under ideal conditions (no noise, no ISI), they are 1 under $\mathcal{H}_{1}$. It can be shown that this is achieved by the normalizations $\tilde{\Lambda}_{k} \triangleq$ $\sqrt{\Lambda_{k} / 122} /\left(16 P_{u}\right)$ and $\tilde{\Lambda}_{k}^{\text {inv }} \triangleq \Lambda_{k}^{\text {inv }} /\left(122 \cdot 16 P_{u}\right)$, where $P_{u} \triangleq$ $\sum_{n=0}^{L-1} \sum_{m=1}^{M}\left|h^{(u, m)}[n]\right|^{2}$. Fig. 5(b) shows the threshold for the normalized statistics, $\eta$, versus the SNR for a detection probability of 0.9 . Here, $\tilde{\Lambda}_{k}^{\text {inv }}$ has a clear advantage over $\tilde{\Lambda}_{k}$ because its threshold is less dependent on the SNR, and thus easier to choose. The corresponding curves for the false alarm probability (not shown) lead to similar conclusions.

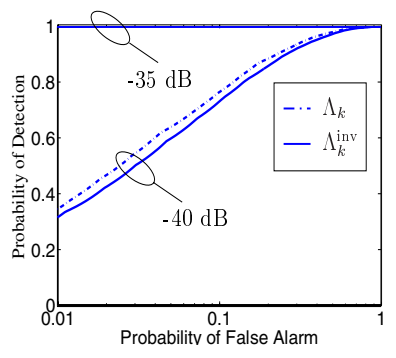

(a)

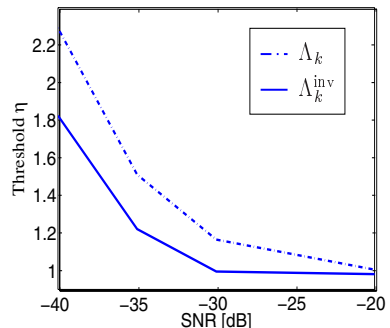

(b)
Fig. 5. Analysis of the detection statistics $\Lambda_{k}$ and $\Lambda_{k}^{\text {inv }}$ : (a) ROCs, (b) threshold $\eta$ vs. SNR for a detection probability of 0.9 .

Even though power control has been disregarded so far, the detection statistics $\Lambda_{k}$ and $\Lambda_{k}^{\text {inv }}$ also work in the presence of power control if the threshold is suitably adjusted. In a TDD system, we have $0.1 \leq c_{k}^{(u)} \leq 1$, corresponding to power control dynamics of $20 \mathrm{~dB}$. It can be shown that under ideal conditions (one BS, no noise, no ISI), the normalized detection statistics $\tilde{\Lambda}_{k}$ and $\tilde{\Lambda}_{k}^{\text {inv }}$ are equal to $c_{k}^{(u)}$. This gives an idea on how to adjust the threshold to be able to detect also the data channels with small gain factors. Note, however, that missing such a weak data channel is not a grave problem because this causes only a small interference for subsequent (weaker) BSs.

\section{Estimation OF GAin FACTORS}

It remains to estimate the gain factor matrix $\mathbf{C}$ or, more precisely, the gain factors $c_{k}^{(u)}$ for all $k \in \hat{\mathcal{L}}^{(u)}$. Because under ideal conditions (one BS, no noise, no ISI) $c_{k}^{(u)}$ equals $\tilde{\Lambda}_{k}$ and $\tilde{\Lambda}_{k}^{\text {inv }}$ as mentioned in Subsection IV-C, we suggest to initially use the estimate $\hat{c}_{k}^{(u)}=\tilde{\Lambda}_{k}$ or $\hat{c}_{k}^{(u)}=\tilde{\Lambda}_{k}^{\text {inv }}$ for detection of the data $\hat{\mathbf{d}}^{(u)}$ of the strongest BS (see Section III). For the subsequent subtraction step (4), however, a more accurate estimate should be used. Indeed, once that $\hat{\mathbf{d}}^{(u)}$ and $\hat{\mathbf{A}}^{(u)}$ are available, we can compute the least-squares estimate

$$
\hat{\mathbf{C}}^{(u)}=\arg \min _{\mathbf{C}}\left\|\mathbf{x}-\hat{\mathbf{A}}^{(u)} \mathbf{C} \hat{\mathbf{d}}^{(u)}\right\|^{2} .
$$

It can be shown that $\hat{\mathbf{C}}^{(u)}=\operatorname{diag}\left\{1, \hat{c}_{5}^{(u)}, \cdots, \hat{c}_{16}^{(u)}\right\} \otimes \mathbf{I}_{122 \times 122}$ with

$$
\hat{c}_{k}^{(u)}=\sum_{l=(k-4) \cdot 122}^{(k-3) \cdot 122-1}\left(\operatorname{diag}\left\{\hat{\mathbf{d}}^{(u)}\right\}^{-1} \hat{\mathbf{A}}^{(u) \#} \mathbf{x}\right)_{l},
$$

where $\hat{\mathbf{A}}^{(u) \#}$ is the pseudo-inverse of $\hat{\mathbf{A}}^{(u)}$.

\section{Simulation Results}

For our simulations, we used Clarke's channel model [11] to simulate a Rayleigh fading channel (see [3] for details). We considered an "outdoor" environment with two different scenarios as illustrated in Fig. 6. In scenario 1, the receiver is located within the inner cell of a grid of 8 hexagonal cells. We will thus encounter one dominant BS signal and 7 weaker BS signals. In scenario 2, the receiver is located at the border of 3 cells, so that there impinge 3 equally strong BS signals 

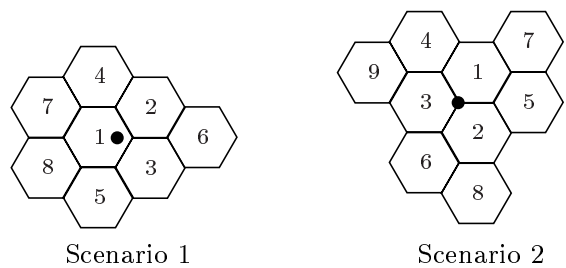

Fig. 6. Simulation scenarios. The bullet $\bullet$ indicates the receiver position.

and 6 weaker BS signals. (This is the more difficult scenario since our DFB receiver assumes only one strongest BS and the other two dominant BS signals act as a strong interference.)

In addition, we used two different channel parameter settings called A and B for each scenario. Channel A has 3 taps with a maximum delay of only 2 chips, whereas channel $\mathrm{B}$ has 8 taps with a maximum delay of 15 chips (thus, channel $\mathrm{B}$ is considerably more challenging than channel A). There were 8 active data channels per BS.

We compared three different detection algorithms, namely,

- the conventional space-time MMSE receiver that estimates

$\mathbf{R}_{\mathbf{x}}$ directly from the received signal x (denoted "MMSE");

- the proposed DFB receiver that uses a space-time MMSE receiver for every BS as explained in Section III (denoted "DFB/MMSE");

- and an extension of the proposed DFB receiver in which the space-time MMSE receiver used for every BS is replaced by the DFB receiver described in [7] (denoted "DFB/DFB").

Table I shows the percentage of successful detection events (i.e., error-free decoding of the $\mathrm{BCH}$ channel corresponding to the respective BS) versus the SINRs $^{1}$ of the various BSs. It is seen that the conventional MMSE receiver performs poorly or even extremely poorly in almost all cases; this is due to the insufficient accuracy of estimating $\mathbf{R}_{\mathbf{x}}$. The proposed DFB/MMSE scheme that uses a calculated (or detected) rather than estimated correlation matrix yields dramatic performance gains even though its computational complexity turns out to be smaller than that of a straightforward implementation of the conventional MMSE receiver. The performance of the $\mathrm{DFB} / \mathrm{DFB}$ scheme is best, however at the expense of some additional complexity compared to the DFB/MMSE scheme.

\section{CONCLUSION}

We proposed a space-time detection algorithm for a UMTS/TDD downlink scenario with strong interference. Because the time variation of the channel does not allow estimation of the input correlation matrix $\mathbf{R}_{\mathrm{x}}$ with sufficient accuracy, we developed a decision feedback scheme that avoids direct estimation of $\mathbf{R}_{\mathbf{x}}$. In each step of the decision feedback scheme, the matrix factors of the correlation matrix corresponding to the (relatively) strongest base station are

\footnotetext{
${ }^{1}$ The definition of the SINR is based on the assumption that the entire component of the received signal corresponding to the BS of interest is the desired signal, i.e., the data channels of this BS transmitted in parallel to the $\mathrm{BCH}$ were not considered as interference.
}

TABLE I

Percentage of successful detection events versus SINR of the various BSs: (a) Scenario 1, (b) scenario 2 .

(a)

\begin{tabular}{|l||c|c|c|c|c|c|}
\hline \multicolumn{1}{|l||}{ Algorithm } & \multicolumn{2}{c|}{ MMSE } & \multicolumn{2}{c|}{ DFB/MMSE } & \multicolumn{2}{c|}{ DFB/DFB } \\
\hline Channel & A & B & A & B & A & B \\
\hline \hline BS 1: $-1 \mathrm{~dB}$ & 100 & 100 & 100 & 100 & 100 & 100 \\
\hline BS 2: $-11 \mathrm{~dB}$ & 34 & 3 & 99 & 99 & 97 & 100 \\
\hline BS 3: $-11 \mathrm{~dB}$ & 26 & 2 & 98 & 99 & 98 & 99 \\
\hline BS 4: $-18 \mathrm{~dB}$ & 1 & 0 & 84 & 84 & 86 & 92 \\
\hline BS 5: $-18 \mathrm{~dB}$ & 0 & 0 & 82 & 88 & 77 & 89 \\
\hline BS 6: $-22 \mathrm{~dB}$ & 0 & 0 & 53 & 52 & 60 & 79 \\
\hline BS 7: $-22 \mathrm{~dB}$ & 0 & 0 & 75 & 57 & 80 & 82 \\
\hline BS 8: $-22 \mathrm{~dB}$ & 0 & 0 & 62 & 57 & 65 & 83 \\
\hline
\end{tabular}

(b)

\begin{tabular}{|l||c|c|c|c|c|c|}
\hline \multicolumn{1}{|l||}{ Algorithm } & \multicolumn{2}{c|}{ MMSE } & \multicolumn{2}{c|}{ DFB/MMSE } & \multicolumn{2}{c|}{ DFB/DFB } \\
\hline Channel & A & B & A & B & A & B \\
\hline \hline BS 1: $-5.1 \mathrm{~dB}$ & 87 & 73 & 99 & 100 & 97 & 100 \\
\hline BS 2: $-5.1 \mathrm{~dB}$ & 85 & 82 & 98 & 99 & 98 & 99 \\
\hline BS 3: $-5.1 \mathrm{~dB}$ & 85 & 65 & 97 & 99 & 99 & 99 \\
\hline BS 4: $-17.1 \mathrm{~dB}$ & 0 & 0 & 62 & 34 & 69 & 59 \\
\hline BS 5: $-17.1 \mathrm{~dB}$ & 0 & 0 & 51 & 45 & 64 & 63 \\
\hline BS 6: $-17.1 \mathrm{~dB}$ & 0 & 0 & 58 & 37 & 71 & 52 \\
\hline BS 7: $-22.1 \mathrm{~dB}$ & 0 & 0 & 9 & 2 & 18 & 12 \\
\hline BS 8: $-22.1 \mathrm{~dB}$ & 0 & 0 & 9 & 2 & 17 & 13 \\
\hline BS 9: $-22.1 \mathrm{~dB}$ & 0 & 0 & 8 & 3 & 14 & 6 \\
\hline
\end{tabular}

determined. An important part of this scheme is the detection of the active data channels, for which a maximal invariant detection statistic was presented. The proposed detection scheme yields good performance at SINRs as low as $-17 \mathrm{~dB}$ for various realistic propagation scenarios.

\section{ACKNOWLEDGMENTS}

The authors would like to thank Gerald Matz and Dominik Seethaler for helpful discussions.

\section{REFERENCES}

[1] http://www.pcrd-antium.com.

[2] K. Kopsa, G. Matz, H. Artés, and F. Hlawatsch, "Space-time synchronisation algorithms for UMTS/TDD systems with strong co-channel interference," in Proc. IEEE Globecom 2002, (Taipeh, Taiwan), pp. 254 258, Nov. 2002.

[3] K. Kopsa, H. Artés, G. Matz, and F. Hlawatsch, "Space-time algorithms for multiuser channel estimation in the downlink of UMTS/TDD," in Proc. IEEE ICC 2003, (Anchorage, AK), pp. 2406-2410, May 2003.

[4] 3GPP, "TS 25.221 Physical channels and mapping of transport channels onto physical channels (TDD)." www.3gpp.org, TS 25.221 v. 4.0.0, March 2001.

[5] 3GPP, "TS 25.223 Spreading and modulation (TDD)." www.3gpp.org, TS 25.223 v. 4.0.0, March 2001.

[6] A. Papathanassiou, Adaptive antennas for mobile radio systems using Time Division CDMA and joint detection. $\mathrm{PhD}$ thesis, Univ. of Kaiserslautern, Germany, 2000.

[7] A. Klein, G. Kaleh, and P. W. Baier, "Zero forcing and minimum mean square error equalization for multiuser detection in code-division multiple-access channels," IEEE Trans. Veh. Technol., vol. 45, pp. 276287, May 1996.

[8] P. Jung and J. Blanz, "Joint detection with coherent receiver antenna diversity in CDMA mobile radio systems," IEEE Trans. Veh. Technol., vol. 44, no. 1 , pp. 76-88, 1995.

[9] S. M. Kay, Fundamentals of Statistical Signal Processing: Detection Theory. Upper Saddle River (NJ): Prentice Hall, 1998.

[10] L. L. Scharf, Statistical Signal Processing. Reading (MA): Addison Wesley, 1991.

[11] R. H. Clarke, "A statistical theory of radiomobile reception," Bell Syst. Tech. J., vol. 47, pp. 957-1000, 1968. 